
\title{
APPLICATION OF 3-D IMAGING IN A FAMILIAL CASE OF
CLEIDOCRANIAL DYSPLASIA
}

\begin{abstract}
Cleidocranial dysplasia (CCD) is a rare inherited disorder affecting dental and skeletal tissues. CCD usually has an autosomal dominant pattern of inheritance and common clinical features seen are aplastic or hypoplastic clavicles, late closure of fontanelle, open skull sutures, retained deciduous teeth, late eruption of permanent teeth and presence of multiple impacted supernumerary teeth. Here, we present a case of CCD in a female patient with positive family history. The diagnosis was confirmed clinically and radiographically. The newer radiographic advancement, $\mathrm{CBCT}$ was used to validate the radiographic findings.
\end{abstract}

Keywords: Cleidocranial dysplasia, impacted teeth, supernumerary teeth, CBCT, CBFA1 gene.

\author{
D Anwesha Biswas ${ }^{1}$ \\ (D) $* \mathrm{G}$ Subhas $\mathrm{Babu}^{2}$ \\ (D) Kumuda Rao ${ }^{1}$ \\ (D) Soundarya Sakthivel ${ }^{1}$ \\ (D) Ananya Madiyal ${ }^{1}$ \\ (i) Renita Castelino ${ }^{1}$
}

ORCID IDs of the authors:

A.B. $\quad 0000-0001-6716-6409$

G.S.B. $0000-0001-9383-7886$

K.R. 0000-0002-6214-1381

S.S. $\quad 0000-0002-3213-9395$

A.M. 0000-0002-0907-3244

R.C. $0000-0002-8696-549 \mathrm{X}$

\begin{abstract}
${ }^{1}$ Department of Oral Medicine and Radiology, A B Shetty Memorial Institute of Dental Sciences, NITTE deemed to be University, Deralakatte, Mangalore.

${ }^{2}$ Department of Oral Medicine and Radiology, NITTE deemed to be University, Deralakatte, Mangalore.
\end{abstract}

How to Cite: Biswas A, Babu GS, Rao K, Sakthivel, Madiyal A, Castelino R. Application of 3-D Imaging in a Familial Case of Cleidocranial Dysplasia. Cumhuriyet Dent J 2020;23:2;142-148.

*Corresponding Author:

Department of Oral Medicine and Radiology, NITTE deemed to be University, Deralakatte, Mangalore.

Phone: +98 $86213571 \quad$ Email: goginenisb@yahoo.co.in 


\section{INTRODUCTION}

Cleidocranial dysplasia is an autosomal dominant bone disease affecting the skeletal and dental system. ${ }^{1}$ Martin in 1765 reported the first case of cleidocranial dysostosis. ${ }^{2}$ CCD also called 'Scheuthauer Marie-Sainton syndrome', 'Marie-Sainton disease' or 'Mutational dysostosis'. CCD affects the bones undergoing intra-membranous ossification, especially skull, clavicles, and flat bones. Hence it is termed as "Cleidocranial dysplasia". It was later found that CCD also affects bones undergoing endochondral ossification and hence, it was said to be a generalized disorder affecting various skeletal structures. ${ }^{3}$ The exact etiology is unknown, but the prime cause in about $20-40 \%$ cases is CBFA1 gene, on chromosome $6 \mathrm{p} 21 .^{4}$ The clinical features of CCD include hypoplasia of mid-face and clavicles giving an appearance of sloping shoulders that can be opposed at the midline; sometimes there may be aplasia of shoulders and abnormalities affecting hands like brachydactyly, short and broad thumbs, tapering fingers. ${ }^{5}$ The CBFA1 gene also plays major role in tooth development and differentiation of ameloblasts and odontoblasts, thus affecting morphogenesis of tooth. ${ }^{7}$ However, in $40 \%$ of cases with $\mathrm{CCD}$, there was no genetic factor. ${ }^{6}$

The dental findings noticed with multiple impacted and supernumerary teeth, are retained primary teeth and delayed permanent teeth eruption resulting in malocclusion. ${ }^{8}$ Features of skull include brachycephalic skull due to delay in ossification of sutures of the cranium and fontanelles; and frontal bossing. Parietal and occipital bones are also affected. Face may appear smaller compared to the cranium. Zygomatic bones and Maxilla are hypoplastic. Paranasal sinuses are also underdeveloped.

With the early diagnosis of CCD, an effective treatment planning can be instituted. Cone-Beam Computed Tomography (CBCT) is a recent advancement in the field of maxillofacial imaging which provides a better 3-D outlook of dentition and skull morphology to the clinician, thus assisting in better treatment planning for the patient. The treatment goal is to give functioning masticatory system to patient and for improved facial appearance. The treatment objective can be achieved with the help of prosthesis, after doing required extractions. The reposition of permanent teeth can be achieved by combination of surgical and orthodontic interventions.

\section{CASE REPORT}

A 8-year-old girl reported to our department with the chief complaint of presence of unerupted teeth in upper and lower jaw since 1 year leading to an unaesthetic facial appearance. She reported delayed eruption of permanent teeth after exfoliation of primary teeth, which led to difficulty in chewing and speech. The patient had the habit of mouth breathing since 2 years. It was also her first visit to a dentist. Medical history revealed that the patient was diagnosed with Cleidocranial dysplasia shortly after birth as per the reports submitted by the patient's parent. Patient was also epileptic and under medication (Carbamazepine $5 \mathrm{mg}$ per day) for the past 4 years. On eliciting family history, patient was a single child and her parents had a nonconsanguineous marriage and father was also a known case of Cleidocranial dysplasia who also gave history of similar complaint in his childhood.

General physical examination revealed that the patient had a short stature and skull was brachiocephalic (Figure 1a).

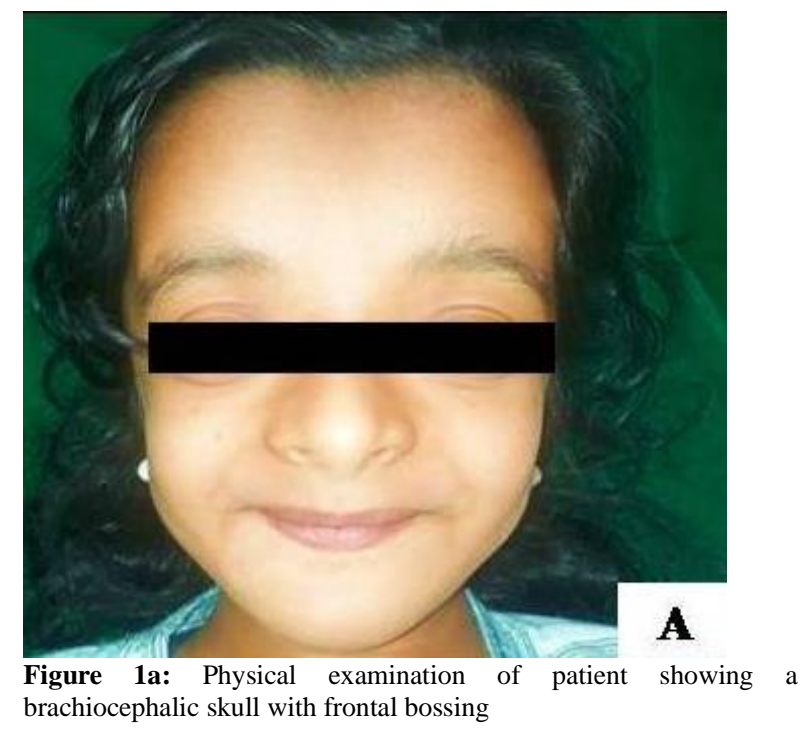

Frontal bossing was evident with a depressed bridge of nose and hypoplastic maxilla and zygomatic arches was underdeveloped resulting in a concave facial profile. On digital palpation, depression was felt over the sagittal suture of 
skull and aplastic clavicles were noted. The patient could approximate her shoulders towards midline (Figure1b).

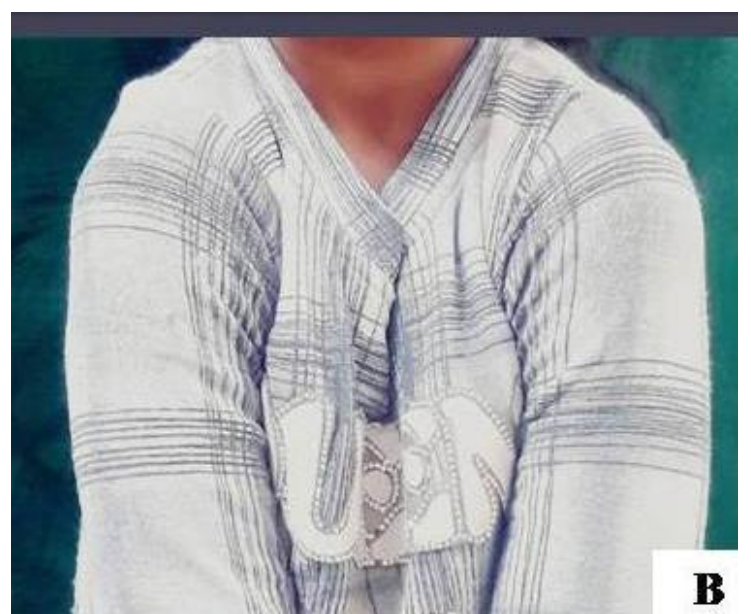

Figure 1b: Approximation of the shoulders to midline to demonstrate absence of clavicles

Extremities showed broad thumbs, brachymetatarsia, overlapping of toes and partial cutaneous fusion of $4^{\text {th }}$ and $5^{\text {th }}$ fingers on right hand of patient (Figure 1c, Figure 1d).

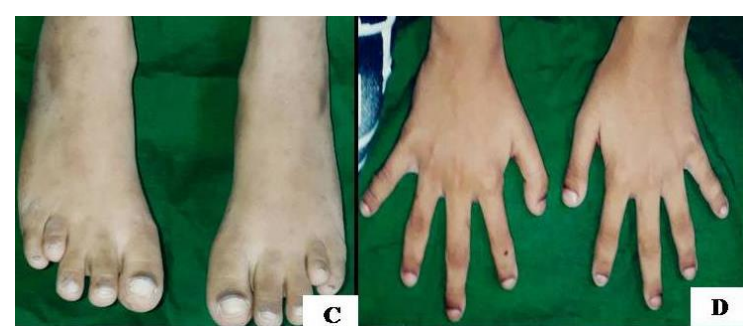

Figure 1c and 1d: Extremities showing broad thumbs and brachymetatarsia.

Intra-orally, hyperpigmentation of dorsum of tongue, high arched palate and generalized gingival inflammation were present. Besides presence of few primary teeth, permanent mandibular lateral incisors were missing. The permanent molars were erupting in both the arches (Figure 2a, Figure 2b). Based on the findings of clinical examination and case history, a provisional diagnosis of Cleidocranial dysplasia was made.



Figure 2a and 2b: Intraoral examination of patient showing hyperpigmentation of tongue, high arched palate and multiple missing teeth
A Panoramic radiograph was made which showed altered bilateral condylar morphology, multiple impacted teeth in the maxillary and mandibular arches, coronal radiolucency with respect to lower deciduous first molar, missing teeth with respect to lower right and left lateral incisor and erupting permanent first molars. Developing permanent tooth buds were seen beneath the primary teeth and supernumerary tooth buds were seen around the permanent tooth buds. (Figure 2c).

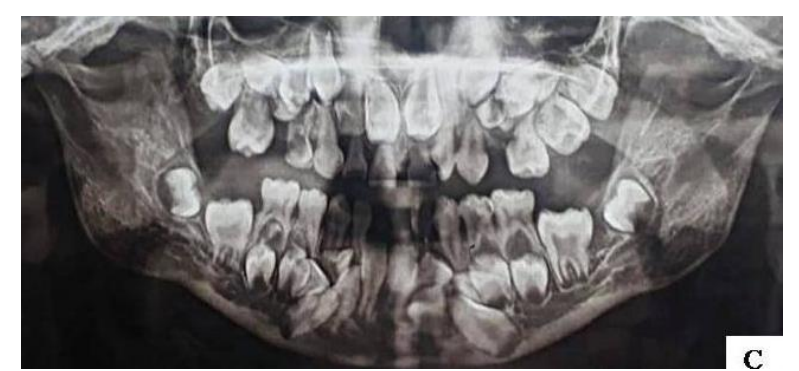

Figure 2c: Panoramic radiograph of patient showing altered condylar morphology, multiple impacted and missing teeth.

The patient was subjected to CBCT, that revealed mixed dentition with multiple impacted supernumerary teeth with respect to maxilla and mandible. Figure $3 \mathrm{a}$ revealed CBCT panoramic reconstruction of patient revealing multiple impacted supernumerary teeth with respect to maxilla and mandible.

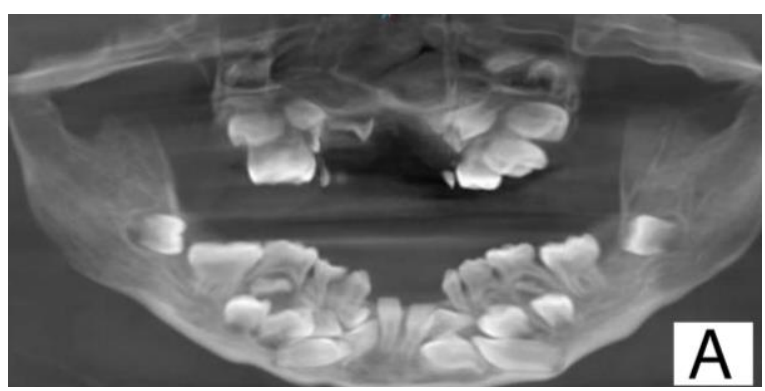

Figure 3a: CBCT panoramic reconstruction of patient revealing multiple impacted supernumerary teeth with respect to maxilla and mandible

Figure $3 b$ and $3 c$ revealed $\mathrm{CBCT}$ anterior view with $3 \mathrm{D}$ reconstruction.

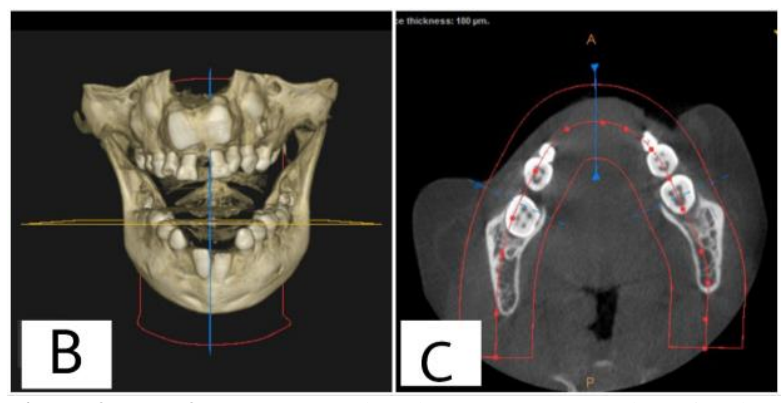

Figure 3b and 3c: CBCT anterior view 3D reconstruction of patient revealing multiple impacted supernumerary teeth with respect to maxilla and mandible. 
Figure $3 \mathrm{~d}, 3 \mathrm{e}, 3 \mathrm{f}$ and Figure $3 \mathrm{~g}, 3 \mathrm{~h}, 3 \mathrm{i}$ shows CBCT of left and right half of the patient's face with $3 \mathrm{D}$ reconstruction and cross sections at every $2.5 \mathrm{~mm}$ respectively.

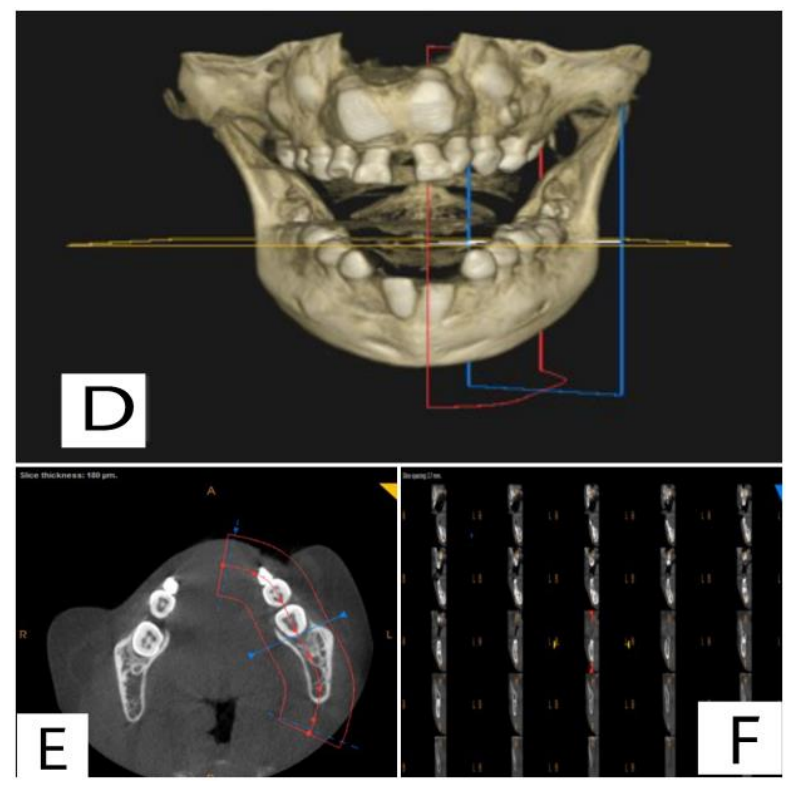

Figure 3d, 3e and 3f: CBCT of left half of the patient's face with $3 \mathrm{D}$ reconstruction and cross sections at every $2.5 \mathrm{~mm}$

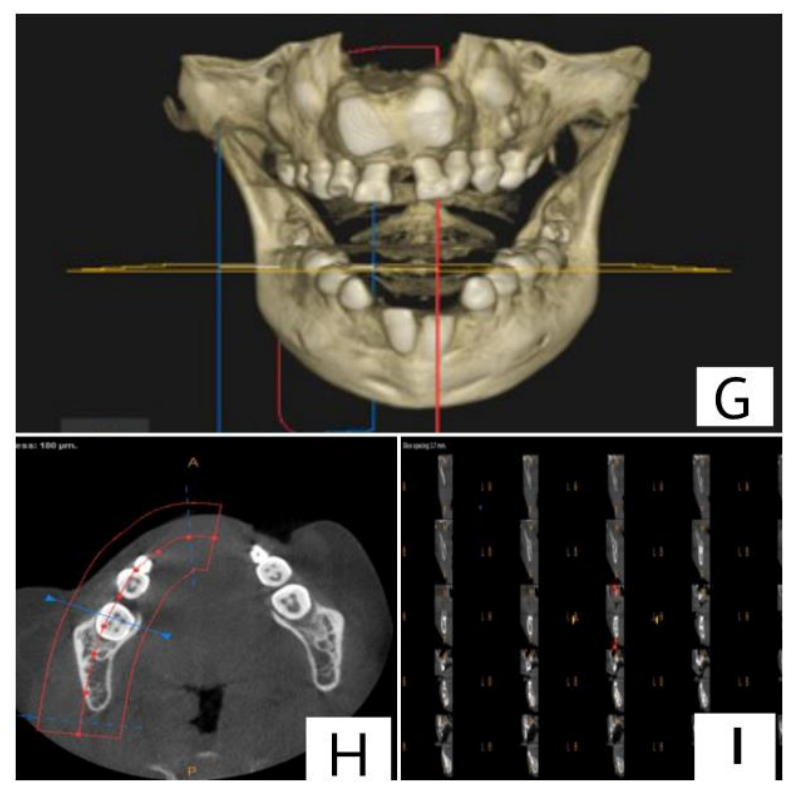

Figure 3g, 3h and 3i: CBCT of right half of the patient's face with $3 \mathrm{D}$ reconstruction and cross sections at every $2.5 \mathrm{~mm}$

No abnormal findings were observed in Hematological investigations. Thus, the diagnosis of Cleidocranial dysplasia was arrived at, with the help of CBCT. A multi-disciplinary approach was planned for a comprehensive management of the patient.

General examination of the patient's father was also made which revealed that he could approximate the shoulders to the midline (Figure $4 a)$.

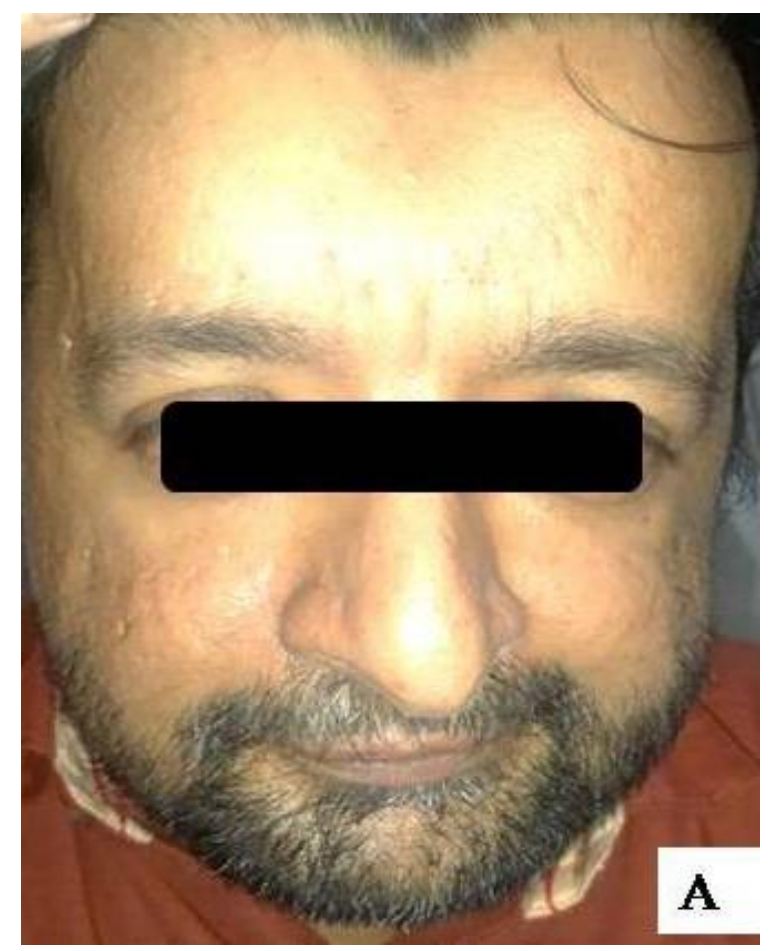

Figure 4a: Physical examination of patient's father showing brachiocephalic skull with frontal bossing

Extra oral examination revealed frontal bossing and brachycephaly (Figure 4b).

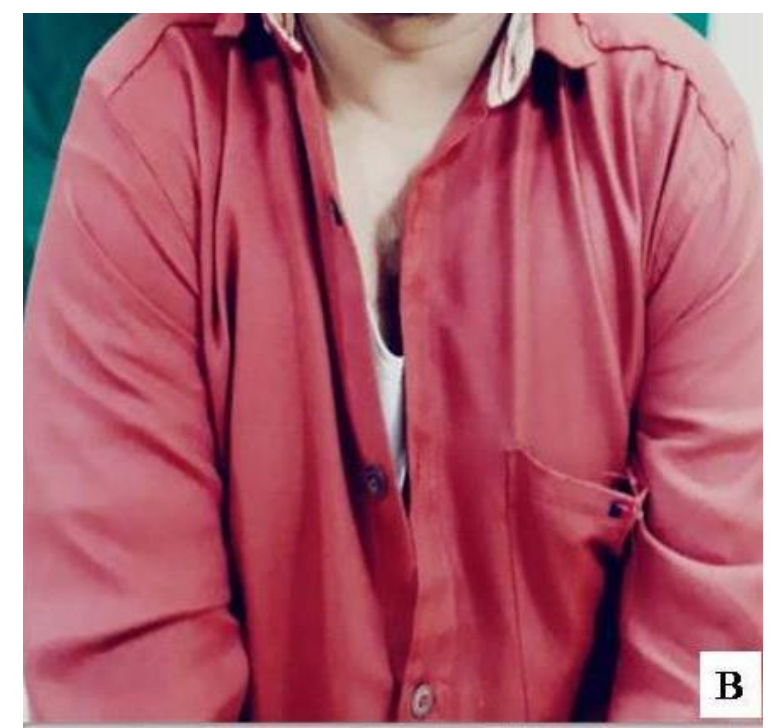

Figure 4b: Patient's father demonstrating approximation of shoulders to midline indicative of missing clavicles

On Intra oral examination multiple anterior and posterior teeth were found missing. Panoramic Radiograph of patient's father revealed altered condylar morphology, multiple impacted teeth in the maxillary and mandibular arches, root stump with respect to upper right maxillary first permanent molar, retained teeth with respect to upper right permanent canine, left first premolar, 
lower left lateral incisor, left canine, right lateral incisor, canine and second premolar and multiple carious teeth with respect to lower left second premolar, second molar and right first molar (Figure 4c).

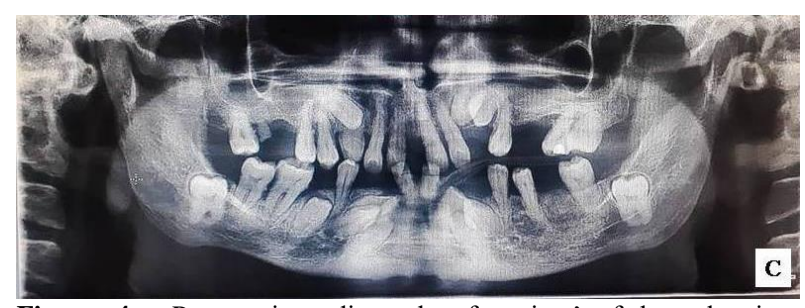

Figure 4c: Panoramic radiograph of patient's father showing multiple impacted and missing teeth.

\section{DISCUSSION}

CCD is commonly misdiagnosed clinically for various other conditions such as Noonan syndrome, Turner's syndrome, hypothyroidism and other skeletal Dysplasia. CCD has a prevalence of 1 per million live births. Its etiology is related to mutation in the gene coding for osteoblast transcription factor Runx2/Core binding factor, which is essential for membranous and endochondral ossification. ${ }^{9}$ The clavicles are hypoplastic, or aplastic, because they are the first bones to ossify. In about $10 \%$ of cases, complete absence of clavicles is observed with affected acromial end. ${ }^{10}$ Maxillary hypoplasia along with mastoid air cells and accessory sinus are also noted. Sometimes, conduction deafness is also noticed in cases of CCD. ${ }^{11}$ The thoracic cage appears to be "bell-shaped" with short and oblique ribs.

Various syndromes and anomalies have similar features as of CCD. Differential diagnosis of CCD includes various skeletal dysplasia like Mandibuloacral dysplasia (MAD), Yunis-Varon syndrome (YVS), Crane-Heise syndrome, Pycnodystosis, Hypophosphatasia (HPP), CDAGS syndrome and osteogenesis imperfecta (OI). Patient anterior fontanelles is a significant feature of CCD as a result of defective ossificiation of Wormian bones in the suture. Similar features can be seen in Pycnodysostosis, Osteogenesis Imperfecta and Congenital Hypothyroidism. Yunis-Varon syndrome is a rare genetic disorder having complete absence of clavicles, similar to $\mathrm{CCD}$, but other manifestations like intellectual disability, abnormalities of hands and feet.
Delayed closure of fontanelles, presence of Wormian bones in sutures. These findings may be seen in other conditions like Pyknodysostosis, Mandibuloacral dysplasia and Yunis-Varon syndrome. Pyknodysostosis is differentiated from CCD. Based on its increased bone density and dwarfism. ${ }^{13}$ Crane-Heise syndrome is another syndrome differentially diagnosed from $\mathrm{CCD}$, by the presence of features like large sized head, mineralized deficiencies of skull, cleft lip and palate, dysmorphic low-set ears, hypoplasia of clavicles and scapulae, abnormalities of cervical vertebrae. Mandibuloacral dysplasia is characterized by short stature, undeveloped mandible and clavicles, delayed closure of cranial sutures. The atrophy of skin with hyperpigmentation, rashes and papular lesions in the trunk and extremities can also be seen. As the age progresses some individuals may develop alopecia. Progressive stiffness of joints may also be noted. Radiograph of fingers and toes reveal acro-osteodysplasia and delayed ossification of carpal bones. CDAGS syndrome is characterized by craniosynostosis, delayed closure of the fontanelles, hypoplasia of clavicles, anal and genitourinary abnormalities, and skin eruption. Hypophosphatasia is characterized by a generalized deficiency in mineralization and ossification. Children affected may exhibit features poorly mineralized bones and widened cranial sutures, short ribs, and a narrow thorax. There is decrease in alkaline phosphatase activity in serum and tissues ..$^{13}$ Due to maxillofacial hypoplasia and osteogenic deficiencies, there is defect in clavicle, cranial and various vertebral and dental abnormalities, Many patients have chest malformations, some severe cases can lead to early respiratory distress in infants and some suffer from hematological disease. Due to osteogenesis and ossification, osteopenia is present in most patients of CCD causing short stature below the two percentiles between 4 and 8 years of age. Although motor development may be delayed, but in most cases mental development is normal. ${ }^{12}$ Intraorally, the presence of supernumerary and impacted teeth in the premolar area is the most important feature. Delayed exfoliation of primary teeth and delayed eruption 
of permanent teeth are other common features. ${ }^{14}$ Similar findings have been noticed in this case report.

Most supernumerary teeth are generally asymptomatic and can be found during routine radiographic examinations, but sometimes they can lead to several pathologies such as tooth displacement, root resorption of the adjacent tooth, cyst formations. ${ }^{15}$ In the present case with impacted supernumerary teeth are common features. Permanent third molars and canines are the most commonly impacted teeth ${ }^{16}$ The etiology behind unerupted teeth is disturbance in resorption of alveolar bone, pre absence of cellular cementum, premature loss of gubernacular canal and disturbance in union between dental follicle and oral mucosa. The abnormalities of skull include multiple Wormian bones, open metopic fontanelles, delayed closure of sutures. ${ }^{14}$

Radiologic investigations play a role important in the diagnostic management of CCD. Various radiographic techniques that are available for the investigation of CCD include Orthopantomogram (OPG), Cone beam computed tomography (CBCT), Micro Computerized tomography (Micro-CT), Computed tomography (CT), etc. Micro-CT methods are recent advancement that allows 3-dimensional measurements of images that are being used in pediatric dentistry. ${ }^{1}$ CBCT is being used in several medical applications, such as angiography, mammography, radiotherapy guidance and it was accepted by the Food and Drug Administration (FDA) in 2001 for the visualization of maxillofacial structures and is being used in almost all areas of dentistry. ${ }^{18}$ Using CBCT, it is possible to obtain sectional images on the axial, sagittal and coronal planes. Various bone abnormalities are best diagnosed on 3D wholebody CT images. After the birth of child, a wholebody radiographic investigation is required for any live-born infant, preterm foetus or stillborn with a suspected constitutional disorder of bone, so that any bony anomaly can be diagnosed at an early stage. ${ }^{19}$ Thus, by a thorough clinical examination and $\mathrm{CBCT}$ radiological examination, a confirmed diagnosis of CCD is obtained.

Management of CCD patient is quite challenging. The prognosis is based on early treatment intervention. ${ }^{20}$ Comprehensive craniofacial management of CCD patient involves the contribution of radiologist, oral maxillofacial surgeon, pedodontist, prosthodontist and orthodontist to provide for better masticatory function and facial aesthetics.

\section{CONCLUSIONS}

The diagnosis of this disorder is often missed or diagnosed at a later age. The patient with CCD often suffers with skeletal handicap and dental, hearing problems and psychological distress. Treatment is based on an interdisciplinary holistic approach by a lineup of dental and medical specialists working as a team.

\section{ACKNOWLEDGEMENTS}

We thank the patients for consenting us to share the details. We also acknowledge Prof. Rohan Fernandez M.A, MBA, HOD Dept .Of Humanities for proofreading the manuscript for English grammar and providing the certificate of the same.

\section{CONFLICTS OF INTEREST STATEMENT}

The authors declare no potential conflicts of interest with respect to the authorship and/or publication of this article.

\section{REFERENCES}

1. More CB, Tailor M, Darjee A, Varma S. Imaging in the diagnosis of a rare anomaly: Cleidocranial Dysplasia. Indian J Oral Sci 2013; 4:86-89.

2. Fernandes DD, Marshall GB, Frizzell JB. Answer to case of the month: Cleidocranial dysostosis. Can Assoc Radiol J 2006; 57:246-248.

3. Manjunath K, Kavitha B, Saraswathi TR, Sivapathasundharam B, Mankandhan R. Cementum analysis in cleidocranial dysostosis. Indian J Dent Res 2008; 19:253-256.

4. Mundlos S. Cleidocranial Dysplasia: clinical and molecular genetics. J Med Genet 1999; 36:177-182.

5. Kerr HD. Cleidocranial Dysplasia. J Rheumatol 1988; 15:359-361. 
6. Dixit R, Dixit K, Paramez AR. Cleidocranial Dysplasia. Lung India 2010; 27:176-177.

7. Chelvan $\mathrm{H} \mathrm{T}, \quad$ Malathi N, Kailasam V, Ponnudurai A. Cleidocranial Dysplasia: A family report. J Indian Soc Pedod Prev Dent 2009; 27:249252.

8. Silva C, DiRienzo S, Serman N. Cleidocranial dysostosis: a case report. Col Dent Rev 1997; 2:26-30.

9. Zhou G, Chen Y, Zhou L, Thirunavukkarasu K, Hecht J, Chitayat D, Gelb B D, Pirinen S, Berry S A, Greenberg C R, Karsenty G, Lee B. CBFA1 mutation analysis and functional correlation with phenotypic variability in cleidocranial Dysplasia. Hum Mol Genet 1999; 8:2311-2316.

10. Golan I, Baumert U, Hrala BP and Mussig D. Early craniofacial signs of cleidocranial Dysplasia. Int J Paediatr Dent 2004; 14:49-53.

11. Visosky AM, Johnson J, Bingea B, Gurney T, Lalwani AK. Otolaryngological manifestations of cleidocranial Dysplasia, concentrating on audiological findings. Laryngoscope 2003; 113:1508-1514.

12. Ma Y, Zhao F, Yu D. Cleidocranial Dysplasia syndrome with epilepsy: a case report. BMC Pediatr 2019;19:1-6.

13. Patil PP, Barpande SR, Bhavthankar JD, Humbe JG. Cleidocranial Dysplasia: A clinico-radiographic spectrum with differential diagnosis. J Orthop Case Rep 2015; 5:21-24.

14. McNamara CM, O'Riordan BC, Blake M, Sandy JR. Cleidocranial Dysplasia: radiological appearances on dental panoramic radiography. Dentomaxillofac Radiol 1999; 28:89-97.

15. Kurt H, Suer T B, Senel B, Avsever H. A retrospective observational study of the frequency of distomolar teeth in a population of 14.250 patients. Cumhuriyet Dent J 2015; 18:335-342.

16. Sener I, Turer A, Bereket C, Ozdemir M. Nonsyndromic familial unerupted teeth: A rare contidion. Cumhuriyet Dent J 2015; 18:359-363.

17. Yuksel B N, Orhan K, Tulga Oz F. Micro-Ct Evaluation of Taurodontism in a Deciduous Molar and a Permanent Molar: Case Report. Cumhuriyet Dent J 2019; 22:486-490.

18. Bayrak S, Sezgin O S, Kayipmaz S, Çan G. The effect of slice thickness on the volume estimations performed by using cone beam CT. Cumhuriyet Dent J 2018; 21:167-172.

19. Tchaou M, Lare P B, Gbande P, Kolou B, Sonhaye L, Agoda-Koussema L K, Adjenou K. Cleidocranial Dysplasia, Radiological Findings in a New-Born (a case report). Int J Radiol 2019; 6:226-228.

20. Gulati S and Kabra M. Cleidocranial Dysplasia. J Postgrad Med 2001; 47: 204-205. 